\title{
A Dental Quality Effort: Sharing the Success Story*
}

\author{
Zurina Abu Bakar \\ Section on Quality in Oral Healthcare, Oral Health Division (Ministry of Health Malaysia), Putrajaya, Malaysia
}

\section{Loh Kim Hong}

Oral Health Division (Kuala Lumpur State Health Department), Kuala Lumpur, Malaysia

\author{
Siti Haniza Mahmud, Samsiah Awang, Nur Ezdiani Mohamed \\ Institute for Health Systems Research (Ministry of Health Malaysia), Shah Alam, Malaysia
}

\begin{abstract}
Mazlina Md Desa
Oral Health Division (Selangor State Health Department, Ministry of Health Malaysia), Shah Alam, Malaysia
\end{abstract}

\begin{abstract}
Quality assurance (QA) was introduced in the oral healthcare program of the Ministry of Health Malaysia for schoolchildren to improve the quality of curative care. One aspect of care monitored was the quality of fillings done on permanent teeth by the dentists and the nurses (the operators). The indicator measured was "percentage of repeat amalgam fillings done on posterior permanent teeth". Following 14 years of monitoring, there was substantial evidence to show that this indicator has been institutionalized among the health personnel involved in the care. Among key factors for success in institutionalizing are top management commitment as well as clear, systematic approach to quality assurance programme. All 15 states in Malaysia showed marked improvement in the achievement of the indicator from 1995 to 2009. Kedah state showed the best achievement (0.04\%) and Melaka state was the least $(0.64 \%)$. This indicator had created awareness among operators of their responsibility to make accurate diagnoses, correct treatment decisions and place quality restorations.
\end{abstract}

Keywords: oral health, indicator, repeat amalgam fillings

\footnotetext{
* The authors would like to acknowledge the Director General of Health, Malaysia, Deputy Director General of Health (Research and Technical Support) and Principal Director of Oral Health Division for the support and the permission to publish this paper. The authors also wish to extend the acknowledgements to all State Deputy Director (Oral Health) and state coordinators for their effort with in ensuring the performance is achieved.

Zurina Abu Bakar, BDS, MCD, Section on Quality in Oral Healthcare, Oral Health Division, Ministry of Health Malaysia, Malaysia; research fields: health management and oral health. E-mail: drzurina.abubakar@moh.gov.my.

Loh Kim Hong, BDS, DDPH, Oral Health Division, Kuala Lumpur State Health Department, Malaysia; research fields: health management and oral health. E-mail: drlohkh.moh@1govuc.gov.my.

Siti Haniza Mahmud, MD, MHP, Ph.D., Institute for Health Systems Research, Ministry of Health Malaysia, Malaysia; research field: health management. E-mail: sitihaniza.m@ihsr.gov.my.

Samsiah Awang, BPharm, MA in Medical Science (Public Health), Institute for Health Systems Research, Ministry of Health Malaysia, Malaysia; research fields: health management and pharmaceutical. E-mail: samsiah241@gmail.com.

Corresponding author: Nur Ezdiani Mohamed, MBBChBAO, Institute for Health Systems Research, Ministry of Health Malaysia; research field: health management. E-mail: nurezdiani.m@ihsr.gov.my.

Mazlina Md Desa, BDS, MCD, Oral Health Division, Selangor State Health Department, Malaysia; research fields: health management and oral health. E-mail: drmazlinamd@moh.gov.my.
} 
The Ministry of Health $(\mathrm{MOH})$ Malaysia as the primary provider, planner, and organizer of medical health and oral health services for the nation is thus the government's lead agency for health. In the MOH, the journey toward institutionalization of quality has always been the paramount goal. This endeavor to ensure optimum quality in healthcare delivery began as early as the 1980s. Since then, numerous initiatives like mortality reviews, infection controls, utilization review, and quality controls were implemented (Hamid et al., 1998).

One of the initiatives founded was the school Incremental Dental Care (IDC) that targeted the schoolchildren. The IDC program that was initiated in 1985 provides a comprehensive and systematic dental care for schoolchildren as the main priority group in the provision of oral healthcare services. The aim was to render schoolchildren orally fit before they leave school. The delivery of care comprises three main components, namely promotive, preventive, and curative care. Since its inception, the IDC program has progressed in strides to provide care in $97 \%$ of primary schools (for children aged 7-12 years old) and 75\% secondary schools (for 13-17 years old), catering for an estimated seven million schoolchildren annually (Division, 2009). This was achieved through the close collaboration with the Ministry of Education, Malaysia.

Parallel to the MOH's goal in ensuring an optimum quality of healthcare delivery, quality assurance (QA) was introduced in the oral healthcare program for the schoolchildren. This was to improve the quality of curative care and to evaluate the outcome of the program. One of the measurements was the quality of fillings done on permanent teeth. In 1995, the indicator "percentage of repeat fillings on anterior and posterior teeth" was monitored as a national indicator with the standard set at 5\%. In 1997, this indicator was differentiated into anterior and posterior repeat fillings to enhance the sensitivity of the indicator (Division, 2006).

From 1995-2009, standards for repeat amalgam fillings done on posterior teeth was progressively revised from 5\% to 1\%. Data from 2007-2009 showed that all states had achieved the set standard. There were many factors contributing to this improved aspect of care. This paper aimed to share the recipe for the successful implementation and institutionalization of this dental quality effort in improving the quality of amalgam fillings on posterior permanent teeth.

\section{Methodology}

Most countries such as European and Middle Eastern countries used outcome measures to improve the quality of care and services delivered by the health care organization to the customer or the patient (Suleiman Hamid, Hussein, Ming, \& Marikar, 2001). The Oral Health Program in the MOH chose a specific outcome, ensuring amalgam fillings done on posterior permanent teeth should last more than five years. It had been established that amalgam fillings formed the bulk of restorations on posterior teeth and there was a high rate of redo fillings. These data were easily retrievable from the Health Management Information System (HMIS). It is known that proper treatment planning, restorative treatment decisions and procedures and good filling materials are fundamental for a lasting filling (Elderton, 1977). Repeat cycles of restoration (filling) can ultimately lead to tooth mortality. The implementation of this indicator would then measure and improve the quality of amalgam fillings on posterior permanent teeth. In the process, the vicious cycle of "restore, remove, and re-restore" and ultimately tooth mortality, is avoided. 


\section{Organizing for Quality}

The indicator "percentage of repeat amalgam fillings done on posterior permanent teeth" was developed based on the consensus of the QA Committee in the Oral Health Division, MOH. It was endorsed for nationwide implementation by the National QA Steering Committee, chaired by the Director-General of Health Malaysia in 1994.

Guidelines for implementation were drawn up to provide guidance in establishing, implementing, and maintaining the QA program (Division, 1992b). These included the terms and definitions of indicators, inclusion and exclusion criteria and reporting formats. The process of gathering information and the personnel responsible for data collection and reporting were also outlined.

The term "repeat fillings" or "redo fillings" was defined as a filling reinserted in the same cavity of a tooth (Division, 1992a). The inclusion criterion was fillings repeated within a period of five years in primary and secondary schoolchildren aged between 7-17 years old. This included fillings repeated due to recurrent caries. Any fillings done by non-MOH staff were excluded.

The data analyzed were repeat amalgam fillings done on posterior permanent teeth from the total number of amalgam fillings done (on posterior permanent teeth) in the current year. The formula is shown in Figure 1. The standard for this indicator was first set at 5\% in 1995.

Percentage of repeat amalgam fillings:

Total number of repeat amalgam fillings done on posterior permanent teeth $\mathrm{x} 100$

Total number of amalgam fillings done on posterior permanent teeth

Figure 1. Formula for indicator percentage of repeat amalgam fillings done on posterior permanent teeth.

\section{Communication}

The guidelines for implementation of this indicator were communicated to all personnel involved at various levels (state, district, and clinic) in the Oral Health Program. The dissemination of information included providing the understanding of the indicator, the inclusion and exclusion criteria, approach for data collection and a manual on how to fill up respective formats. In ensuring quality, data were collected, training and standardization on diagnosis of dental caries (calibration) was carried out on a regular basis. In addition, clinical audits were conducted at random.

All 15 states in Malaysia were involved in the implementation of this indicator. Process of gathering information involved reviewing dental treatment cards and reporting formats from individual primary and secondary schools. These data were later aggregated monthly from the schools to another format developed at national level.

\section{Monitoring and Evaluation}

Monitoring was done at clinic (monthly), district (monthly), at state (six monthly) and at national level (six monthly and yearly). Dedicated personnel were assigned to ensure complete data were collected at each of these levels and reported as scheduled. Investigation for non-performers was initiated at the district and state level and remedial measures were devised and implemented.

The National Oral Health QA Committee Meeting was chaired by the Principal Director of Oral Health, $\mathrm{MOH}$. With the State Deputy Directors (Oral Health) as members of the committee, they reviewed QA reports, 
studied trends and made recommendations regarding revision of optimum achievable standards. Annual and half-yearly QA reports presented at these meetings were disseminated to all states and districts, allowing for learning and sharing of remedial actions and good practices. These reports were also presented to the QA National Technical Committee and QA National Steering Committee, MOH.

\section{Results}

The indicator "percentage of repeat amalgam fillings done on posterior permanent teeth" went through 14 years of implementation at three different standards. Based on the performance of the various states, the standard was reviewed and adjusted from $5 \%$ to $2 \%$ in 1998 . This new standard was consistently achieved from 1998-2002. After three consecutive cycles, the standard was further refined to $1 \%$ in 2003 . Table 1 shows the continual improvement of the standard.

Table 1

Changes in the Standard of the Indicator From 1995 to 2009

\begin{tabular}{llll}
\hline Indicator & \multicolumn{2}{l}{ Standard } & \\
\cline { 2 - 4 } & $1995-1997$ & $1998-2002$ & $2003-2009$ \\
\hline $\begin{array}{l}\text { Repeat amalgam fillings done on posterior } \\
\text { permanent teeth }\end{array}$ & $\leq 5 \%$ & $\leq 2 \%$ & $\leq 1 \%$ \\
\hline
\end{tabular}

Generally, there was a declining trend from 1995 to 2009 (see Figure 2). There was a sharp drop in "repeat fillings" from 1995 (4.6\%) to 1998 (1.7\%). However, the improvement was more gradual from 1.7\% to $0.29 \%$ between 1998 and 2009. By 2009, all the states in Malaysia had achieved the standard set of $1 \%$ (see Figure 2).

Marked improvement of the indicator was achieved in all states from 1997 to 2009, with the state of Kedah showing the best achievement $(0.04 \%)$ and Melaka the least $(0.64 \%)$. Two states which were consistently lower than others were Sabah $(0.45 \%)$ and Melaka $(0.64 \%)$. Pahang and Perak reported a drastic improvement from $3.0 \%$ in 1997 to $0.05 \%-0.06 \%$ respectively in 2009 (see Figure 3).

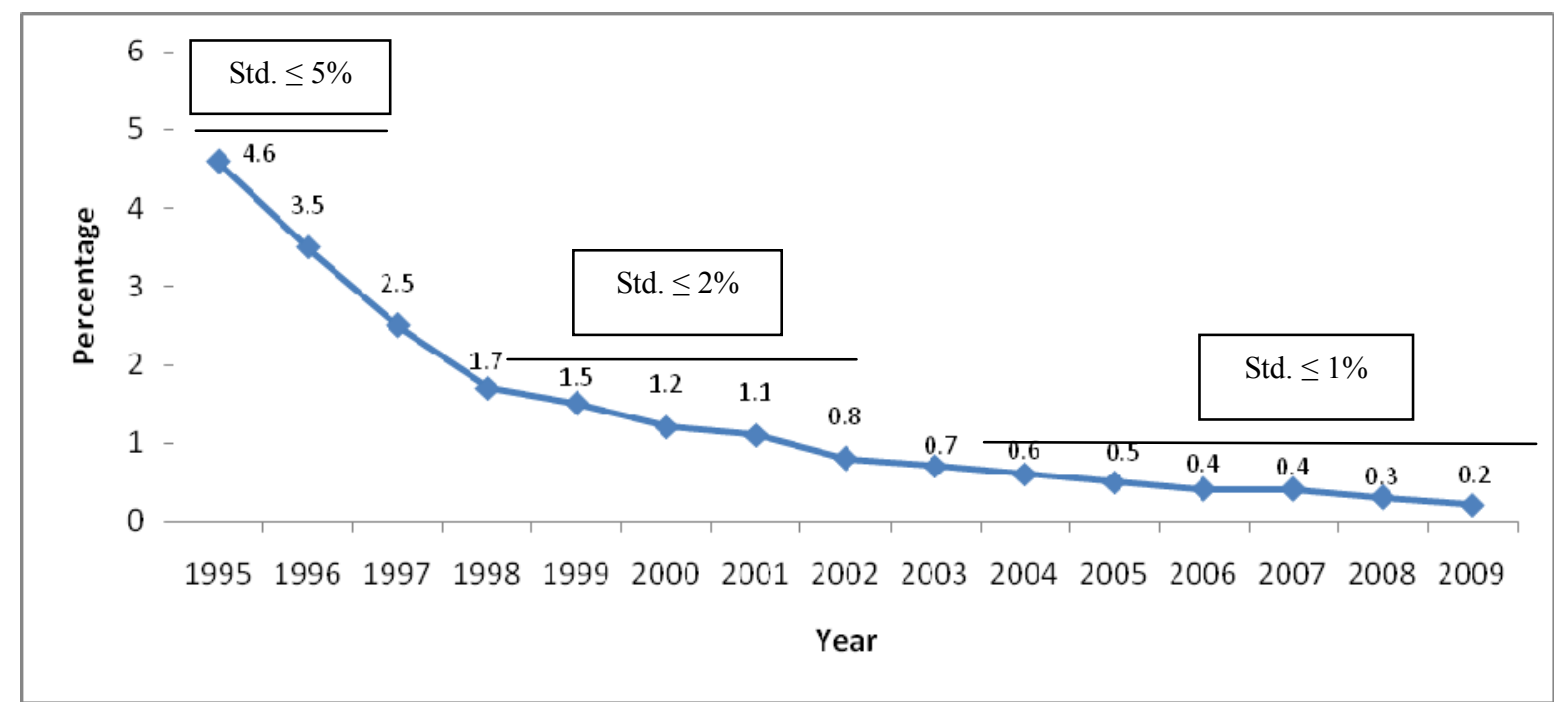

Figure 2. Achievement of the indicator percentage of repeat amalgam fillings done on posterior permanent teeth (1995-2009). Source: Oral Health Division, MOH (2009). 


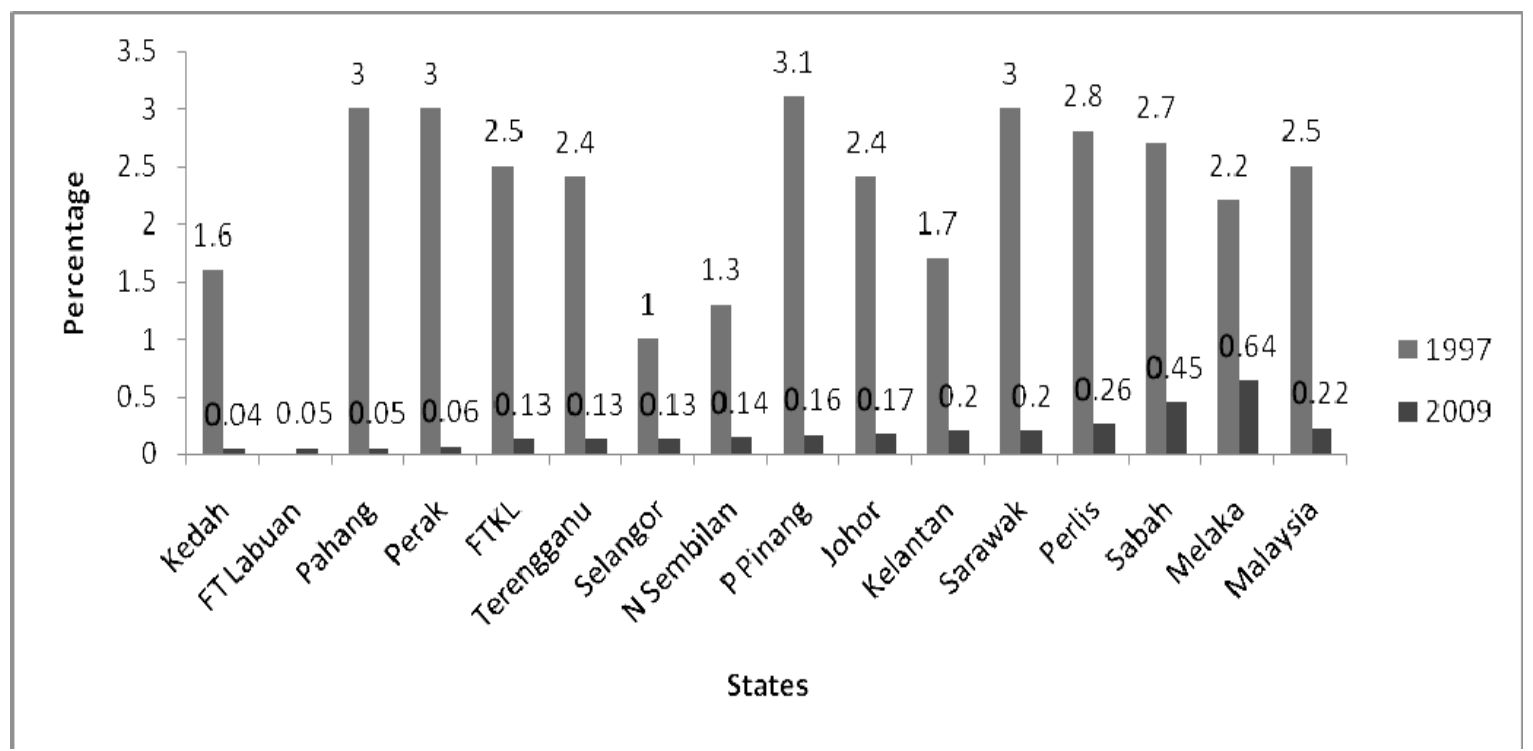

Figure 3. Comparison of achievement of the indicator percentage of repeat amalgam fillings done on posterior permanent teeth (1997 \& 2009). Source: Oral Health Division, MOH (1997 and 2009).

\section{Discussion}

Institutionalization requires a clear delineation of roles and responsibilities and accountability for the implementation of QA activities. The National QA Committee, headed by the Principal Director of the Oral Health Program, MOH was formed to develop its QA program. The early phase included the identification of indicators, the search and selection of the appropriate standards for adoption. Similar committees or personnel responsible were appointed at state and district level for effective implementation and monitoring.

Another factor that had contributed to the successful implementation of this indicator was training and supervision. At the onset of implementing the indicator, training was given to health staff and managers creating a climate of awareness and acceptance of the concept and a commitment in using QA as a management tool to improve the quality of healthcare delivery. Briefing and training of the various personnel involved in data collection were carried out. Inaccurate and incomplete data or late submissions of reports were immediately tackled at the state level with the involvement of staff at district/s level. The appointment of dedicated personnel helped put these in check.

In addition, health personnel were taught to identify factors contributing to shortfalls in quality and to devise and implement appropriate remedial measures. In doing so, indicators which were not achieving the standards were addressed in a timely manner and remedial measures were put in place. For example, one district in Pahang found weaknesses in the lack of attention to procedures involved in placing amalgam restorations (80\%) and knowledge of staff $(60 \%)$. Another district in Selangor attributed the poor achievement in the indicator to improper tooth preparation, poor handling of amalgam and inadequate post-operative advice to patients.

In most states, training and re-training, in the form of Continuing Dental Education (CDE) conducted as in-house training, was the most common remedial measure. Standardization and calibration exercises in caries diagnosis and treatment needs are scheduled on a regular basis, as part of the school IDC program. These often involve new dental nurses and officers. In areas where the turnover of oral health personnel were high or where it was called for as a remedial measure, calibration exercises were carried out on a more regular basis. 
Other training included updates of case management emphasizing current concepts of disease and prevention and current concept of cavity preparation. This is supported by studies which have indicated that measures improving the oral health maybe of major importance in preventing recurrent caries and thereby increase the durability of amalgam restorations. There is a clear need to move toward a truly preventive form of caries management, with the placement of modern restorations in high quality conservative cavity preparations on a selective basis (Elderton, 1994).

The concepts and theories learned were also translated into the "Model of Good Care (MOGC) for amalgam filling on posterior teeth" in which oral health instructions that could help the patient in controlling the disease process were included. These instructions could be incorporated at any level of the MOGC such as in the conduct of tooth brushing drills and dental health education for schoolchildren during visit to schools by the dental team.

Monitoring the quality of amalgam fillings in the school IDC program has a far-reaching effect in reducing tooth mortality among schoolchildren. It is noteworthy that reducing the number of repeat amalgam fillings may help in reducing the exposure of the patient, dental personnel and the environment to mercury, as it is known that small amounts of mercury are released from amalgam during placement and removal (Federation Dentaire Internationale, 2007; Mitchell, Koike, \& Okabe, 2007).

Looking at the factors mentioned earlier, there were closely linked with the indicator in place. The achievement of the indicator will trigger the investigation alarm and thus the following remedial measures. Thus, interpretation of the result must be done in careful manner taking into consideration other confounding factors. As seen in the early phase of the indicator's implementation, initial optimum achievable standard of less than 5\% repeat amalgam fillings on posterior permanent teeth that was based on review of HMIS records may seem high compared with findings in studies (Letzel, Hof, Marshall, \& Marshall, 1997; Soncini, Maserejian, \& Trachtenberg, 2008) in which the annual failure rates of amalgam fillings ranged from $0.16 \%$ to $2.83 \%$ and a replacement rate of $10.8 \%$ and repair rate of $0.4 \%$ were found in permanent teeth in a 5 -year follow-up. These findings served as mere comparisons, bearing in mind that they were results of clinical trials and the same fillings which were followed through. On the other hand, the 5\% standard from HMIS records was derived from the formula of number of repeat amalgam fillings over the total number of amalgam fillings placed in the current year. It involved different cohorts of children and fillings, thereby lacking the scientific rigor of controlled prospective evaluations.

The sharp annual drop of about one percent annually in the first three years of implementation (1995-1998) should be interpreted with caution, as this could mean under-reporting of repeat fillings. This was especially so when non-independency of examiners existed due to the inherent nature of the IDC program, where schools were assigned to each dental team. Dental nurses and officers tend to screen the same children every year and this modus operandi might have continued into the early phase of this indicator.

In 2002, pre-capsulated alloy was introduced in the Oral Health Services in Malaysia, phasing out the use of bulk alloy. It could have contributed to the continued improvement in performance of the indicator from 2002-2009, as evidenced by a study where alloy selection significantly influenced the survival of the restorations for reasons directly related to the restoration (Letzel et al., 1997).

By 2009, all states had already achieved the standard of $1 \%$ for three consecutive years. The Oral Health QA Committee considered it unrealistic to achieve zero defects since there were factors beyond control; for example, the role and responsibility of the patient in complying with home care instructions. Based on strong 
evidence that this indicator had been institutionalized, the committee made the decision to remove it as a national indicator. However, states were recommended to adopt it as an indicator in districts that were still lagging behind. By that time, there was already a critical mass of staff, with the awareness, understanding, and skills to work on their own at district level.

Without doubt, adequate resources (human resource, facilities, and financial allocation) and effective resource management were the key elements that led to the successful outcome of the implementation of this indicator. In addition, a well-designed QA policy and effective leadership were essential elements contributing to the institutionalization of QA, as shown in Table 2.

Table 2

Essential Elements for the Institutionalization of QA

\begin{tabular}{|l|l|l|}
\hline Internal enabling environment: & Organizing for quality: & Support functions: \\
\hline $\begin{array}{l}\text { Policy } \\
\text { Leadership } \\
\begin{array}{l}\text { Core values } \\
\text { Resources }\end{array}\end{array}$ & Structure & $\begin{array}{l}\text { Capacity building } \\
\text { Information \& communication } \\
\text { Rewarding quality }\end{array}$ \\
\hline
\end{tabular}

\section{Limitations and Challenges}

Examiner bias could have led to under-reporting of repeat amalgams. Random checks through clinical audits and rotation of operators to schools each year were done to overcome or minimize this. There were cases of lost records, especially when students changed schools and dental records were left behind or lost. This posed a problem for operators to access past dental history. For continuity of care, cooperation from the schools is important to ensure that dental records follow the student, when he/she goes on transfer.

\section{Conclusions}

The existence of the QA indicator "percentage of repeat amalgam fillings done on posterior permanent teeth" since 1995 has created an awareness among operators of their responsibility to make accurate diagnoses, correct treatment decisions and place quality restorations, within the context of a preventive form of caries management. Being monitored through the National Indicator Approach, it has been found to be a very useful management tool at all levels in the Oral Health Program. These efforts have led to better quality fillings through the various off-shoots of quality improvement initiatives and have been well institutionalized after 14 years.

\section{References}

Division, O. H. (1992a). Dental HMIS manual. Ministry of Health.

Division, O. H. (1992b). Guidelines for implementation of NIA. Ministry of Health.

Division, O. H. (2006). Annual Report of Oral Health Division. Ministry of Health.

Division, O. H. (2009). Annual Report of Oral Health Division. Ministry of Health.

Elderton, R. (1977). The quality of amalgam restorations. In H. Aired (Ed.), Assessment of the quality of dental care (pp. 45-81). London: London Hospital Medical College.

Elderton, R. (1994). Critical look at operative dentistry. Annals of Dentistry, 1, 5-9.

Federation Dentaire Internationale, P. S. (2007). Safety of dental amalgam. FDI General Assembly Dubai.

Hamid, M. A., Low, P., Abdullah, A. R., Yon, R., Morad, N., Hussein, R. H., ... Bakar, M. A. A. (1998). The strategic plan for quality in health. Kuala Lumpur: Percetakan Asni Sdn. Bhd.

Letzel, H., Hof, M. V. T., Marshall, G., \& Marshall, S. (1997). The influence of the amalgam alloy on the survival of amalgam restorations: A secondary analysis of multiple controlled clinical trials. J Dent Res, 76(11), 1787-1798. 
Mitchell, R., Koike, M., \& Okabe, T. (2007). Posterior amalgam restorations-Usage, regulation, and longevity. Dental Clinics of North America, 51, 573-589.

Soncini, J., Maserejian, N., \& Trachtenberg, F. E. A. (2008). The longevity of amalgam versus compomer/composite restorations in posterior primary and permanent teeth: Findings from the New England Children's Amalgam Trial. J Public Health Dent, 68, 14-21.

Suleiman, A. B., Hamid, M. A., Hussein, R., Ming, D. L., \& Marikar, M. A. K. (2001). Quality assurance in Malaysia. In A. F. Al-Assaf (Ed.), Health care quality: An international perspective. New Delhi, World Health Organization. 